\title{
Personal training by vaccination
}

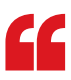

feasibility
and potential
clinical success
of vaccines

targeting multiple neoantigens in patients with melanoma

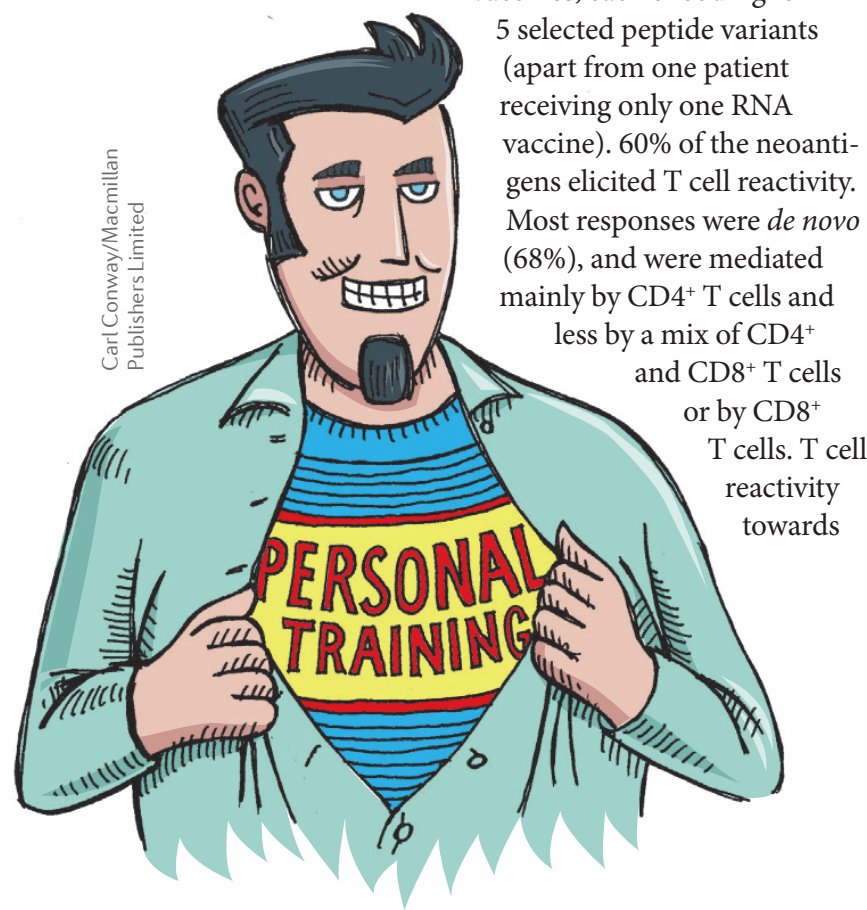

The heterogeneity of cancer makes individualized treatment options necessary, even in immunotherapy. Although neoantigens can prompt spontaneous tumour-specific immune responses, these are mostly insufficient to stop tumour growth. Two groups have now independently demonstrated that, through the use of personalized neoantigen vaccines, $T$ cell responses can be efficiently triggered and outcomes improved in patients with melanoma.

Sahin et al. used an RNA vaccine approach. They identified tumour-specific, non-synonymous mutations in 13 patients with stage III and IV melanoma through exome-sequencing and RNAsequencing. Mutations encoding for peptide variants with high predicted binding to human leukocyte antigen (HLA) class I and II molecules were selected. The researchers injected each patient with two synthetic RNA vaccines, each encoding for 5 selected peptide variants (apart from one patient receiving only one RNA ) $60 \%$ of the neoantigens elicited $\mathrm{T}$ cell reactivity. Most responses were de novo (68\%), and were mediated inly by $\mathrm{CD} 4^{+} \mathrm{T}$ cells and and $\mathrm{CD}^{+} \mathrm{T}$ cells by $\mathrm{CD} 8^{+}$ T cells. T cell reactivity wards wards neoantigen-loaded dendritic cells was non-existing or weak pre-vaccination and detectable or augmented post-vaccination, as well as highly specific. Following vaccination, all 13 patients showed significantly reduced cumulative recurrent metastatic events and sustained progression-free survival. Out of these, eight patients that presented with no detectable lesions pre-vaccination remained completely free of melanoma relapses. Five patients that presented with lesions pre-vaccination showed complete (two patients, one of whom required combination treatment with anti-programmed cell death 1 (PD1) antibodies), partial (one patient, showing initial response but later on progressed probably owing to resistance conferred by $\beta_{2}$-microglobulin loss) or mixed (one patient) responses, or stable disease (one patient). Importantly, vaccination led to increased PD1 ligand 1 (PDL1) expression in some tumours. Following anti-PD1 antibody treatment post-vaccination, one patient, still showing fast disease progression post-vaccination, experienced a complete response. Therefore, patients could benefit from combining neoantigen vaccination with PD1 blockade.

Ott et al. used a peptide-based vaccine approach. They identified tumour-specific somatic mutations and selected 13-20 mutations per patient, based on the predicted ability of the encoding peptide to bind to autologous HLA class I molecules. For each neoantigen, a clinical grade immunizing long peptide was synthesized, pooled in 4 groups and given to the patient. Interestingly, even though peptides had been selected for HLA class I binding, most immune responses, only detected post-vaccination, were prompted by $\mathrm{CD} 4^{+} \mathrm{T}$ cells $(60 \%)$ and less by a mix of $\mathrm{CD}^{+}$and $\mathrm{CD} 8^{+}(10 \%)$ or $\mathrm{CD}^{+} \mathrm{T}$ cells (16\%). More than $30 \%$ of the $\mathrm{CD}^{+}$and $\mathrm{CD} 8^{+} \mathrm{T}$ cells was polyfunctional, and their neoantigen reactivity was highly specific. Moreover, gene expression profiling revealed that post-vaccination, neoantigen-specific $\mathrm{CD} 4^{+} \mathrm{T}$ cells clustered separately from pre-vaccination $\mathrm{CD} 4^{+} \mathrm{T}$ cells and showed elements of effector and memory functions. Six of the ten initially enrolled patients, presenting with previously untreated, high-risk melanoma (stage III or stage IV), received the vaccination. Four patients, who had presented with stage IIIB/C melanoma, showed complete responses and no disease recurrence post-vaccination, whereas the two patients who had presented with stage IV melanoma showed disease recurrence. However, following treatment with anti-PD1 antibodies, these two patients showed complete responses. In these patients, the number of neoantigens that elicited $\mathrm{CD}^{+}$or $\mathrm{CD}^{+} \mathrm{T}$ cell responses was increased after anti-PD1 treatment.

Together, these two studies demonstrate the feasibility and potential clinical success of vaccines targeting multiple neoantigens in patients with melanoma. This approach, in combination with PD1 checkpoint blockade, will hopefully benefit patients with late-stage cancer with high mutational load, for whom treatment options are very limited.

Ulrike Harjes

ORIGINAL ARTICLES Sahin, U. et al. Personalized RNA mutanome vaccines mobilize poly-specific therapeutic immunity against cancer. Nature $\mathbf{5 4 7}$ 222-226 (2017) |Ott, P. A. et al. An immunogenic personal neoantigen vaccine for patients with melanoma. Nature 547, 217-221_(2017) FURTHER READING Yarchoan, M. et al. Targeting neoantigens to augment antitumour immunity. Nat. Rev. Cancer 17, 209-222 (2017) 